\title{
Planification Du Temps Scolaire Sur Les Performances Des Élèves Du Primaire Dans L'extrême-Nord Du Cameroun
}

\author{
Djara Ndjidda* \\ PhD student at the Faculty of Education Sciences (Management of Education III) at the University of Yaoundé \\ 1, Cameroon. \\ *Corresponding Author: Djara Ndjidda, PhD student at the Faculty of Education Sciences \\ (Management of Education III) at the University of Yaoundé 1, Cameroon.
}

\begin{abstract}
Schoolchildren in Extreme-North Cameroon experience harsh climates on their school times and rhythms in the afternoons, periods of high heat. Thus, this article demonstrates that school time influences the realities constituted by the child's rhythm. The latter take into account his age, his geographic environment, even per-extracurricular times, etc. With this, the aim of this research aims to better program the lessons at favorable times for these students. Moreover, it is a question of identifying the difficulties relating to their retention and the use of the teachings of the schools of the said region. Also, it is necessary to propose the strategies which can be adopted to improve the conditions of learning. The approach followed is mixed. The interview and the questionnaire were the data collection tools for this research. The population was made up of directors, teachers, parents and students. It therefore appears that the planning of school time in this region is not in line with the aforementioned realities. The learning of many students is disrupted during periods of very hot weather. Hence the problem of failure or even dropping out of school. as a suggestion, this research proposes the abolition of half-time, the abundant construction of classrooms, the flexibility of school youth and the massive recruitment of qualified teachers in school spaces in the far North in general and the region of Extreme-North in particular.
\end{abstract}

Key words: planning, school time, school performance, climatic hazards, rhythms.

\section{INTRODUCTION}

Sur le plan national, les écoles primaires au Cameroun fonctionnent selon trois régimes :

à plein-temps : c'est-à-dire toutes les classes de l'école sont fonctionnelles de lundi, mardi, jeudi et vendredi de $07 \mathrm{~h} 30$ à $15 \mathrm{~h} 00$.

- $\quad$ à mi-temps : les groupes des classes (de la SIL au CM2) sont opérationnelles du lundi au vendredi en matinée de $07 \mathrm{~h} 30$ à $12 \mathrm{H} 30$ et, d'autres groupes de $12 \mathrm{~h} 3017 \mathrm{~h} 30$ ou éventuellement les samedis.

à mixte : certaines classes fonctionnent à plein temps tant dis que d'autres à mi-temps (informations du rapport PASEC (Programme d'Analyse des Systèmes Educatifs de la CONFEMEN en 2014).

Dans le cadre de la semaine de 05 jours, l'enseignement dispensé aux élèves du primaire actuellement est réparti sur 180 jours de classes. Autrement dit 36 semaines de 05 jours correspondant à 1104 heures de cours annuels et 34 heures 30 minutes de volume hebdomadaire pour le plein temps. Le mi-temps est réparti quant à lui sur 853 heures 20 minutes du volume annuel et 26 heures 40 minutes du volume hebdomadaire au niveau I (de la SIL au CP). Au niveau II (CE1-CE2), le volume annuel est de $862 \mathrm{heures} 30$ minutes et le volume hebdomadaire est de $34 \mathrm{~h} 30 \mathrm{~min}$ pour le plein-temps et, $687 \mathrm{~h} 30 \mathrm{~min}$ de volume annuel et $25 \mathrm{~h}$ de hebdomadaire pour le mi-temps. Face à cette segmentation chronologique, les élèves du primaire de ladite région, en des périodes des hautes chaleurs, sont confrontés aux problèmes extrêmement complexes. A titre illustratif, nous énumérons des effectifs pléthoriques, le matériel d'enseignement non conforme, une mauvaise maitrise des programmes du système à mi-temps, les ressources matérielles obsolètes. De plus, on assiste à la survenue de quelques problèmes cruciaux concernant la réussite scolaire de ces élèves. Ce sont autant 
de facteurs qui viennent accroitre le poids de l'insuffisance d'organisation ou de la médiocrité du niveau des élèves sur leur évolution.

Par ailleurs, les fonctions physiologiques de l'être-humain sont liées à des rythmes biologiques d'environs 24 heures: rythmes circadiens. Chaque individu a une " horloge biologique interne " propre avec l'alternance de deux états: veille et sommeil (Cermakian, 2000 ; Sheer, 2005). Cependant plusieurs facteurs déterminent ces rythmes. D'une part, les facteurs endogènes déterminés par un centre régulateur situé au sein d'une petite structure appelée « Noyau Suprachiamatique », qui intervient dans la sécrétion d'une hormone: la Mélanie. D'autre part, les facteurs exogènes ou externes dont les déclencheurs sont l'environnement (lumière, humidité, température, salinité des mers etc.) et les phénomènes cosmiques (alternance jour-nuit, phases de lune, variation de saison, etc.). Les facteurs externes agissent sur l'organisme à travers son système nerveux. Ceux-ci ne créent pas les rythmes, ils ne font que les moduler. On les appelle synchroniseurs ou agents entrainants, ou encore agents donneurs du temps (Zeigeber). Ce sont eux qui activent normalement le système endocrinien. Les synchroniseurs prépondérants chez l'homme sont de nature socio-écologique et sont représentés par les alternances lumière-obscurité et repos-activité et des facteurs sociaux tels que les horaires des repas, du sommeil voir (Touitou, 1998a). Tous ces indicateurs ont besoin d'être mieux managés afin de permettre à l'élève de s'adapter dans les processus d'apprentissage.

L'enseignement primaire dans la région de l'Extrême-Nord connait une difficulté structurelle, organisationnelle mais aussi une situation économique précaire. Les études démontrent que la manière dont les jeunes utilisent leur temps libre est largement conditionnée par le milieu. La nature et la qualité des activités extrascolaires pratiquées par les jeunes influent sensiblement sur leurs chances de réussite scolaire (Bonnérry, 2006). L'enfant arrive fatigué à l'école avec une tête et un corps qui chauffent à $12 \mathrm{~h} 00$ quel que soit le type de travail qu'il a eu à effectuer avant de s'y rendre, ou quel que soit la durée de son repos ou de son sommeil la nuit précédente, puis va augmenter progressivement ses capacités d'attention et d'apprentissage dans l'après-midi accompagné d'un climat rude. Ceci génère chez les enfants les plus fragiles une fatigue excessive, voire épuisante. Mais aussi la souffrance, le stress chez les petits enfants qui sont les plus vulnérables. La démotivation qui à son tour entraine le plus souvent l'absentéisme aux cours, le manque de ponctualité, qui pourrait conduire à l'abandon de l'école qui est un autre grave problème affectant plusieurs sociétés et communautés Africaines en générale et le Cameroun en particulier, il s'agit d'identifier les difficultés relatives à l'apprentissage et à l'utilisation des enseignements des écoles de ladite région. Aussi, faut-il proposer des stratégies idoines pouvant être adoptées pour améliorer les conditions des apprentissages des enfants. Les résultats scolaires de ces dernières années et les niveaux des apprenants sont en baisses dans cette région. Quelles sont les causes de cette baisse ? Les conditions climatiques sévères suivant les périodes de l'année, perturbent -elles les temps et rythmes scolaire des élèves du primaire dans la région de l'Extrême Nord du Cameroun ? Les variations annuelles des performances scolaires des élèves sont-elles fonctions des variations climatiques ? Comment l'administration scolaire gère-t-elle les activités péri et extrascolaire dans l'Extrême-Nord Cameroun ? Comment le personnel enseignant prend-il en compte les données des élèves pour un rendement optimal en dépit des rudes conditions climatiques ? L'objectif de cette étude vise à mieux programmer les enseignements afin de solutionner ce problème.

\section{Presentation de La Zone D'etude Et Description Du Climat De La Region}

\subsection{Présentation De La Zone D'étude}

La région de l'Extrême-Nord du Cameroun est située entre le 10ème et 13ème degré de latitude nord et du 13ème au 15ème degré de longitude Est. Elle s'étire sur près de $325 \mathrm{~km}$, des pays soudanais jusqu'aux improbables rivages du lac Tchad. Elle est limitée à l'Est par le Nigéria, à l'ouest par le Tchad, et au Sud par la région du Nord Cameroun (ATLAS, 2000). C'est la deuxième région la plus peuplée du pays après le Centre pour une densité moyenne de 101,6 habitants/ km2. Avec une population estimée à 3480414 habitants au 1er Janvier 2010, soit 17,9\% de la population totale du Cameroun. La région de l'Extrême Nord a pour chef-lieu Maroua et c'est une unité administrative qui comprend six départements à savoir :

- $\quad$ Le Diamaré qui a pour chef-lieu Maroua et dispose de 09 arrondissements ; 
- $\quad$ Le Mayo-Danay dont Yagoua est le chef-lieu et dispose de 11 arrondissements ;

- $\quad$ Le Mayo-Kani qui a pour chef-lieu Kaélé et dispose de 07 arrondissements ;

- $\quad$ Le Mayo-Sava, dont Mora est le chef-lieu et se compose de 03 arrondissements ;

- Le Mayo-Tsanaga qui a pour chef-lieu Mokolo, le département le plus peuplé de la région avec 07 arrondissements ;

Le Logone et chari qui a pour chef-lieu Kousseri avec 10 arrondissements.

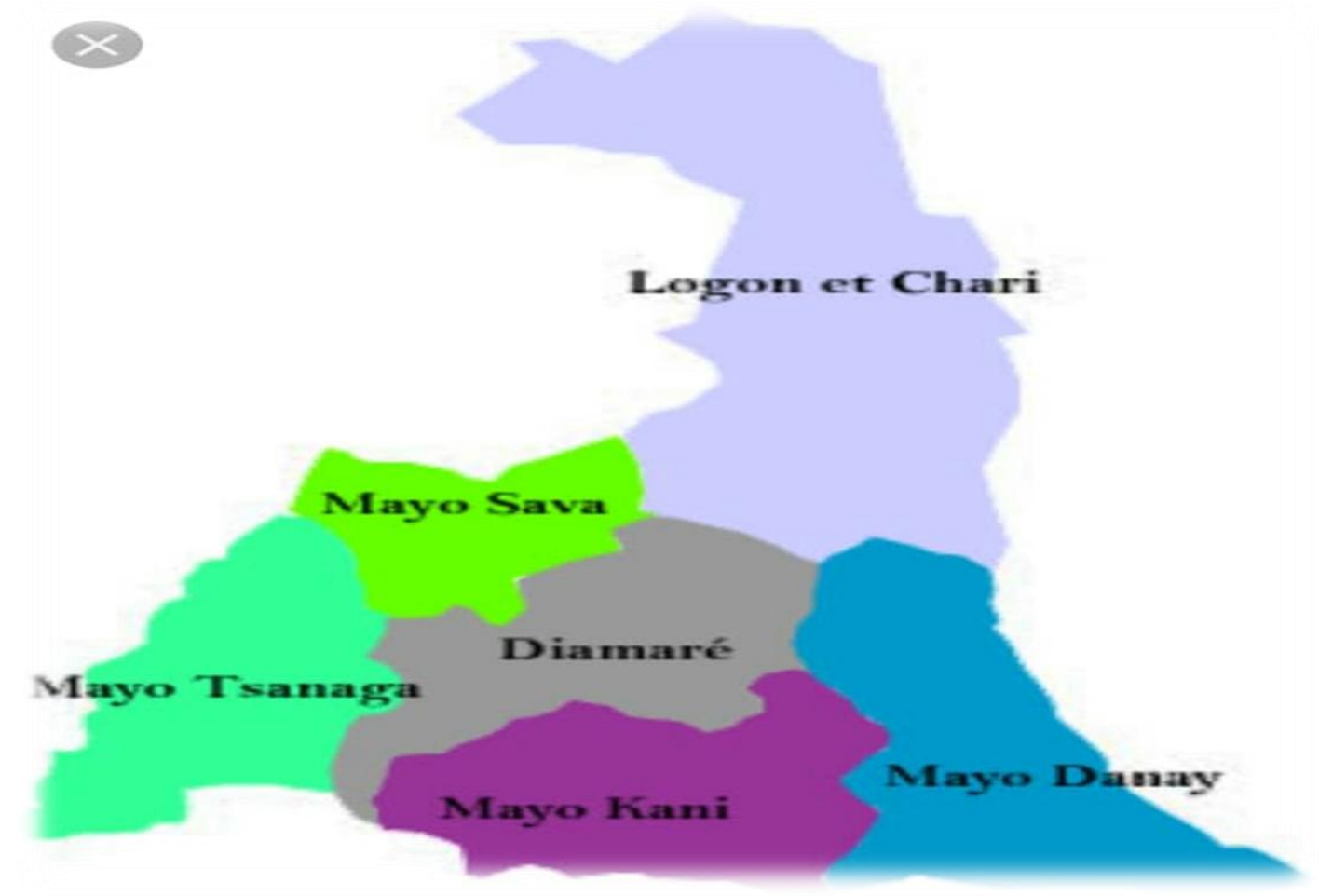

Source: Atlas géographique 2000 in Wikipédia.org.

\subsection{Description Du Climat De La Région De l'Extrême-Nord}

Son climat est de type Soudano-sahélien à deux saisons : une saison sèche de 8 à 9 mois et une saison de pluies de 3 à 4 mois. Les précipitations y sont assez faibles avec une moyenne annuelle de 800 $\mathrm{mm}$. Globalement, cette zone est soumise à un climat tropical au sens large dont les principales caractéristiques sont:

Une insolation importante et des températures élevées oscillant en moyenne entre 30 à $50^{\circ} \mathrm{C}$;

* Une seule saison de pluie qui va de juin à fin octobre ;

Une saison sèche d'autant plus rigoureuse et longue qui va de novembre à mai.

Toute fois le mois d'août est plus pluvieux. La température est généralement très élevée qui varie entre 18 et $42^{\circ} \mathrm{C}$. Généralement on note trois grandes périodes de variation thermiques : une période de froid ; une période de chaleur et une période tempérée. La totale pluviométrie est de 900 millimètres de pluie répartis en 60 jours pour une température moyenne de quelques $28^{\circ} \mathrm{C}$ :

\section{. Hydrographie}

L'hydrographie est composée de quelques fleuves et des cours d'eaux qui prennent leurs sources dans le fleuve Logone.

\section{- Végétation.}

Cette végétation est composée principalement des mimosacées constituées des différentes variétés d'acacias, des césalpiniacées, des papilionacées, des rôniers. C'est une végétation de steppe arborée parsemée des plantes épineuses naines et d'herbes parasites. Les principales espèces florales qu'on trouve sont le savonnier, le tamarinier etc... 


\section{. Faune.}

Elle est composée des oiseaux migrateurs, des petits gibiers tels que les écureuils et les lapins. On y peut ajouter les rongeurs, les souris et les rats qui y prolifèrent. D'autres espèces d'oiseaux y vivent également à savoir les vautours, les mange-mil, les éperviers, les pique-bœufs, les pigeons, les corbeaux, les aigles, les pintades, les hérons, etc. La faune aquatique quant à elle regroupe les hippopotames, les varans et une grande variété d'espèces poissonneuses (le tilapia, la carpe, le machoiron, le silure, le capitaine, etc.) Mais au-delà de cette richesse faunique, on note la menace considérable de disparition de certaines espèces du fait de la chasse, des feux de brousse et de la déforestation, voire de la rudesse du climat.

\subsection{Impact Du Climat Sur L'éducation Des Elèves Dans La Région De l'Extrême Nord}

Les facteurs climatiques ont plusieurs impacts directs et indirects sur l'éducation des enfants. Un climat plus chaud modifie l'aire de répartition géographique, vecteurs de plusieurs maladies telles que le paludisme, la fièvre typhoïde etc... Et à ces facteurs, il faut ajouter l'action de l'homme à travers la déforestation, les feux de brousse, le braconnage etc...l'impact du changement climatique se fera sentir à plusieurs niveaux par des perturbations dans la possibilité d'aller en classe : c'est déjà le cas dans ces régions, où en des périodes de grosses chaleurs, les élèves les plus fragiles ne peuvent pas aller à l'école dans les après-midis.

\section{Probleme De L'étude.}

Les fonctionnements des écoles dans cette région sont relatifs à la situation géographique, financière, organisationnelle et culturelle. Cependant, dans les zones périurbaines, on rencontre des écoles bien loties en bâtiments servant de classe à l'instar de celles des centres urbains. Toutefois, l'une des différences les plus remarquables à n'en point douter concerne les effectifs des élèves très pléthoriques en ville et très faible en campagne. A ce sujet, Hilaire sikounmo cité par M. Ndogmo « dans l'éducation scolaire et lien social » (2006:69) avance même des chiffres qui alarment : «au Cameroun on retrouve couramment des classes du primaire de plus de 150 élèves. » Il faut également mentionner une sous scolarisation, l'insuffisance d'infrastructures de communication et l'insuffisance d'infrastructures socioéconomiques. Le mobilier (bancs, bureau) qui remonte à une date relativement lointaine n'est guère réjouissant. La qualité et la quantité ont un impact négatif sur leurs propres performances (encadreurs) et par ricochet sur celles des élèves. Dans ces écoles où la pénurie des enseignants a pour conséquence entre autres le jumelage des cours dans certaines zones rurales. Sur 04 enseignants titulaires interrogés dans l'école publique de Dougoi (Maroua 3ème), tous les 02tiennent des classes jumelées. Dans une de ces écoles, nous avons rencontré une enseignante qui cumulativement avec son titre de directrice, tient à elle seule les deux cours de son école, les classes de CM2. Dans ce cadre, il est difficile de dispenser un enseignement de qualité surtout avec la rudesse climatique. On note aussi le manque d'accès pour ces enfants aux commodités de la vie moderne d'aujourd'hui tels que des aires de jeu aménagés, de l'eau potable, les toilettes propres, aussi une bibliothèque fournie etc....

Sur le plan qualitatif, la situation du personnel est approximative du fait de manque d'enseignants selon la qualification professionnelle. On rencontre des instituteurs de l'enseignement maternel et primaire, des Maitres d'Enseignement Général Adjoint (MEGA), et des maitres bénévoles qui n'ont aucune formation mais qui ont appris à enseigner dans le tas selon leur rémunération.

Sur le plan quantitatif, il y a un sureffectif d'enseignants dans les salles de classe de nos grandes villes. Pourtant, dans nos zones rurales, le manque d'enseignants se fait sentir de manière criarde.

La préoccupation dans cet article ne se fonde pas seulement sur une critique de la planification des temps scolaires au niveau primaire, mais il est question de démontrer en quoi l'organisation du temps scolaires plus adéquate avec les facteurs environnementaux et climatiques sont nécessaires pour les jeunes écoliers. Il faut que la question du temps et rythme scolaire soit en adéquation directe avec d'autres aspects essentiels de l'éducation comme celui du traitement de la difficulté scolaire, et plus généralement encore celle de l'efficacité des pratiques d'enseignement, (Atttali, Bressoux, 2002). Il est donc intéressant de mettre en évidence les travaux liés à la problématique de l'organisation du temps et aux rythmes d'apprentissage des élèves du primaire selon certaines spécificités locales (situation humaine, climatique, situation économique, physique, psychologique...). 
Les écoles primaires de cette région vivent des conditions matérielles difficiles (à cause de l'absence des moyens multiformes : enseignants mal payés ou pas du tout, absence des moyens d'encadrement des élèves etc...). A cela s'ajoute l'influence directe et négative de plusieurs facteurs environnementaux tels : les saisons, les insomnies, le manque d'électricité. Or, le temps scolaire en lui-même est normé, il est organisé sur la base d'un continuum d'objectifs mesurables ; quantifiables ; administrables, il est découpé, planifié ; rythmé selon les évaluations ; des cycles réguliers ; répétitifs Perrenoud (2012) ; Tardif et Lessard, (1999).

\section{Mthodologie De L'ETUde}

La démarche empirique ici est centrée sur l'analyse du rôle de la planification des temps sur les performances des élèves du primaire dans l'Extrême -Nord du Cameroun.

\section{1.Échantillonnage Et Démarche De Collecte Des Données.}

Les données empiriques proviennent de l'enquête effectuée par l'auteur auprès de cent cinquanteneuf (159) individus repartit ainsi qu'il suit : élèves (61); délégué à l'éducation (02); inspecteurs de l'enseignement primaire (03). parents d'élèves(43); enseignants des salles de classes(50) rencontrés au sein des écoles primaires dans la région de l'Extrême-Nord du Cameroun susceptible de fournir des informations importantes et nécessaires servant à la réalisation de la recherche constitué respectivement de 101 hommes $(63,5 \%)$ et 58 femme $(36,5 \%)$.

la collecte de données s'est faite en deux phase: une recherche documentaire qui comprend la consultation des ouvrages, des articles, des documents officiels ainsi que tous ceux qui ont un lien direct ou indirect avec notre travail afin de mieux comprendre notre sujet et bénéficier des études précédentes et le questionnaire qui a été administré directement des individus dans la région de l'Extrême-Nord du Cameroun, susceptible de fournir des informations importantes et nécessaires servant à la réalisation de la recherche.

Ce questionnaire a permis de recueillir des informations relatives à l'analyse du rôle de la planification des temps sur les performances des élèves du primaire dans l'Extrême- Nord Cameroun. La technique non probabiliste nous a permis d'effectuer un échantillonnage non aléatoire par la méthode des quotas. Et les acteurs sont connus d'avance et peuvent faire partie des services différents ou alors être dans le même service et aussi parce que la méthode des quotas est celle la plus répandue du fait qu'en pratique, elle donne de bon résultats, à condition d'avoir été pratiqué avec méthode et rigueur.

Nous avons opté dans cette étude pour une recherche mixte parce que, loin de rendre les deux approches imperméables l'une à l'autre, le fait qu'elles renvoient à deux domaines d'intelligibilité fonde leur complémentarité (Couty, 1984). Winter (1984:1) partage ce point de vue lorsqu'il signale que l'une et l'autre constituent des " méthodes d'investigation irréductibles mais complémentaires ». On note d'ailleurs, au sein de la communauté scientifique, la volonté d'opérer une synthèse entre analyses qualitative et quantitative.

Toutefois, pour mettre en œuvre des systèmes d'investigation prenant en compte simultanément des méthodes qualitative et quantitative, il est nécessaire au préalable d'identifier les enjeux méthodologiques sous-jacents afin de penser un appariement qui conserve à chacune son identité propre. C'est à cette condition que la combinaison entre approches qualitatives et approches quantitatives permettra d'enrichir et d'affiner notre compréhension.

En accord avec ces propos, toute combinaison de techniques de collecte de données Qualitatives et Quantitatives est possible en méthode mixte. Les chercheurs en méthode mixte sont créatifs et utilisent toutes les formes de collectes de données expérimentales, observationnelles ou simulées. Néanmoins, les techniques de collecte traditionnelles prédominent. Le questionnaire structuré tend à prédominer du côté des techniques Quantitatives, et l'entrevue ouverte ou semi-structurée tend à prédominer du côté des techniques Qualitatives (Bryman, 2006). Certaines techniques de collecte de données Qualitatives et Quantitatives méritent une mention spéciale, car elles constituent un élément important dans l'identification et la sélection des méthodes mixtes qui servent à tester nos hypothèses.

Les deux approches ne s'opposent donc pas. Elles se complètent: L'approche qualitative, par observation, par entretien, par protocoles (etc....) permet de récolter énormément d'informations 
(Couty, 1984). Certaines d'entre elles n'étaient pas attendues. Elles font progresser la recherche. Cependant la durée d'une enquête qualitative limite son recours à des sujets de recherche pour lesquelles on dispose de peu d'informations. L'enquête qualitative sera choisie dans une phase exploratoire d'un nouveau sujet de recherche. Elle permet de développer une théorie et relève donc d'un processus inductif. Cependant ce qui fait la force de l'approche quantitative (profondeur des entretiens) est source de faiblesses (durée de l'entretien) : on ne peut interroger qu'une faible partie des individus. La validité externe de la recherche est questionnable (Couty, 1984).

Nous avons fait usage de design de complémentarité de la méthode mixte parce qu'il est question pour nous de prendre en compte différents niveaux d'analyse d'un même phénomène afin de mieux comprendre le problème de recherche et en plus, la complémentarité permet de mesurer différentes facettes d'un phénomène afin d'en obtenir une compréhension plus riche. l'intention pour nous dans cette recherche c'est de bénéficier des différents avantages des méthodes qualitatives (plus de détails, de profondeur) à travers un guide d'entretien administré aux directeurs d'écoles et les enseignants rencontré dans les bureaux à l'inspection et à travers un questionnaire destiné aux élèves ; délégué à l'éducation; inspecteurs de l'enseignement primaire; parents d'élèves ; enseignants des salles de classes rencontrés au sein des écoles primaires dans la région de l'Extrême-Nord du Cameroun susceptible de fournir des informations importantes et nécessaires servant à la réalisation de la recherche réduisant ainsi les faiblesses de chacune par la complémentarité de l'autre.

Ensuite nous avons fait usage d'un processus simultané, dans lequel nous souhaitons rapprocher les données quantitatives et qualitatives afin de fournir une analyse complète de la question de recherche. Dans cette conception, les deux formes de données ont été recueillies en même temps et ont ensuite été intégrées dans l'interprétation des résultats globaux.

Ainsi, dans le cas de notre étude, nous optons pour la méthode qualitative basée sur des entretiens individuels semi-directifs à travers une approche qualitative inductive parce que la méthode qualitative s'intéresse à la signification des phénomènes. Cette stratégie de recherche empirique paraît la plus apte à répondre concrètement à la problématique étudiée. L'étude de cas vise à utiliser un ou des exemples réels afin de faciliter la compréhension et la connaissance en répondant aux questions «comment» et «pourquoi» permettant ainsi de tirer plus facilement des enseignements au sujet du phénomène observé. Dans la logique phénoménologique, le monde est socialement construit et subjectif. Dès lors, la science est indissociable des intérêts humains.

C'est pourquoi le chercheur doit s'intéresser à la signification des phénomènes et déduire ses idées à partir des données elles-mêmes. C'est dont l'aspect qualitatif des données qui importe et non l'aspect quantitatif. Pour saisir cet aspect, il faut procéder par induction, c'est-à-dire laissé parler le terrain. Cette approche nous semble spécifiquement indiquée pour aborder l'étude de «la planifications sur les performances des élèves du primaire dans l'Extrême-Nord Cameroun». Nous nous situons dans cette section dans une logique de découverte et de description pouvant conduire à une construction théorique et non dans une logique explicative ou encore quantitative.

L'approche qualitative permet l'obtention d'informations dans un secteur où rien ou quasi rien n'est connu au sujet du problème étudié et permet la conceptualisation, étant d'une grande flexibilité ainsi que l'adaptation aux théories nouvelles et innovantes. Et dans cet ordre d'idée qualitative, l'on s'intéresse aux individus/personnels ressources à savoir: les directeurs d'écoles (06) et les enseignants(15) rencontrés au sein des écoles primaires dans la région de l'Extrême-Nord du Cameroun susceptible de fournir des informations importantes et nécessaires servant à la réalisation de la recherche.

Nous avons retenu l'analyse de contenu qualitative parce que non seulement ce type d'analyse s'intéresse au fait que des thèmes, des mots ou des concepts soient ou non présents dans un contenu. L'importance à accorder à ces thèmes, mots ou concepts ne se mesure pas alors au nombre ou à la fréquence, mais plutôt à l'intérêt particulier, la nouveauté ou le poids sémantique par rapport au contexte. Et enfin dans cette partie de la recherche, l'on a fait usage de la triangulation des outils de cueillette qui renvoie au fait de faire usage de plus d'un outil (par exemple, utiliser des entrevues, des observations, de l'analyse de documents). 


\subsection{La Méthode D’analyse Des Données De L'analyse Quantitative.}

à partir des données collectées, les méthodes de traitement et d'analyse des données suivantes ont été utilisées : l'analyse descriptive sur l'échantillon qui permettra d'analyser les données récoltées par le questionnaire; la méthode interprétative qui consiste à faire des tableaux et des graphiques et tirer des conclusions et les perspectives au regard des résultats de l'étude afin de proposer des recommandations adaptées ; l'analyse statistique qui consiste à faire des tests statistiques en vue de les confirmer ou d'infirmer les hypothèses formulées dans le cadre du travail. Cette analyse fera usage du test de Coefficient de corrélation de Pearson (R).

En effet Nous allons fait usage du test de coefficient de corrélation de Pearson (R) et non des modèles économétriques parce que non seulement notre variable dépendante (ou phénomène étudiée) qui est ici la planification des temps scolaires sur les performances des élèves de la région de l'Extrême-Nord est un phénomène purement qualitatif (non chiffrable) dans la vie courante de tous les jours et surtout elle est appréciée à travers des indicateurs qualificatifs que sont : le moment où l'enfant se couche le soir à la maison, le temps que met l'enfant pour rentrer à la maison, le moyen de locomotion qu'emprunte l'enfant pour rentrer de l'école etc...

L'analyse des données outre la description des fréquences, des moyens et la description de celle-ci, comprend une description des corrélations simples ou d'ordre zéro (c'est-à-dire où aucun contrôle de l'influence des autres variables n'est effectué entre chacune des variable ou encore, une description des corrélations partielles (c'est à dire où les effets d'une variable ou de plusieurs variables additionnelles sont pris en considération) entre deux variables. Dans le présent article, nous avons procédé à un échantillonnage par les quotas dans les classes et en dehors des classes.

Il s'est agi de tirer une portion de la population de telle manière que chaque membre y ait une chance égale d'être sélectionné. Ce procédé est considéré comme le meilleur moyen de sélectionner un échantillon représentatif. La technique de traitement et d'analyse des données dans cet article dépend de la nature des variables, du modèle de recherche et des hypothèses de recherche. Nous avons eu recours à l'enquête auto administrée qui se justifie par sa capacité à offrir la possibilité au répondant de prendre tout le temps qu'il juge nécessaire pour lire le questionnaire, le comprendre et le remplir posément et en plus l'enquête auto administrée permet d'éclaircir aux enquêtés certains éléments qui pouvaient leur sembler obscurs, mais aussi et surtout pour les rassurer quant à l'usage de l'information demandée et nous avons obtenu un taux de réponse au questionnaire définie comme le rapport entre nombre de répondants effectifs et nombre de répondants espéré au départ dont l'ordre est de $90,85 \%$.

\subsection{Les Logiciels Utilisés Dans La Démarche De L'analyse Quantitative.}

Dans le cadre de cet article, nous avons eu recours à deux logiciels différents à savoir: SPSS (Statistical Package for the Social Sciences) version 20 noté simplement SPSS.20 pour le dépouillement du questionnaire et les autres tests utiles est enfin EXCEL version 7 noté simplement EXCEL.7 pour les graphiques. Dans les lignes qui suivent, nous présentons les principaux résultats de l'étude.

\section{Presentation Des Resultats}

Il est question dans cette sous-section de présenter l'analyse uni varié de quelques résultats de l'enquête (5.1) et l'analyse bi variée de quelques résultats de l'enquête (5.2).

\subsection{Analyse Uni Varié De Quelques Résultats De L'enquête.}

Il est question ici de présenter l'analyse descriptive sur les différentes saisons observées, les variations annuelles des performances scolaires, la satisfaction de l'adoption des réformes locales en matière d'éducation et enfin les récapitulatifs de certains variables des conditions climatiques sévères dans la région de l'Extrême -Nord.

\subsubsection{Différentes Saisons Observées Dans La Localité De l'Extrême -Nord Actuellement.}

Graphique : différentes saisons observées 


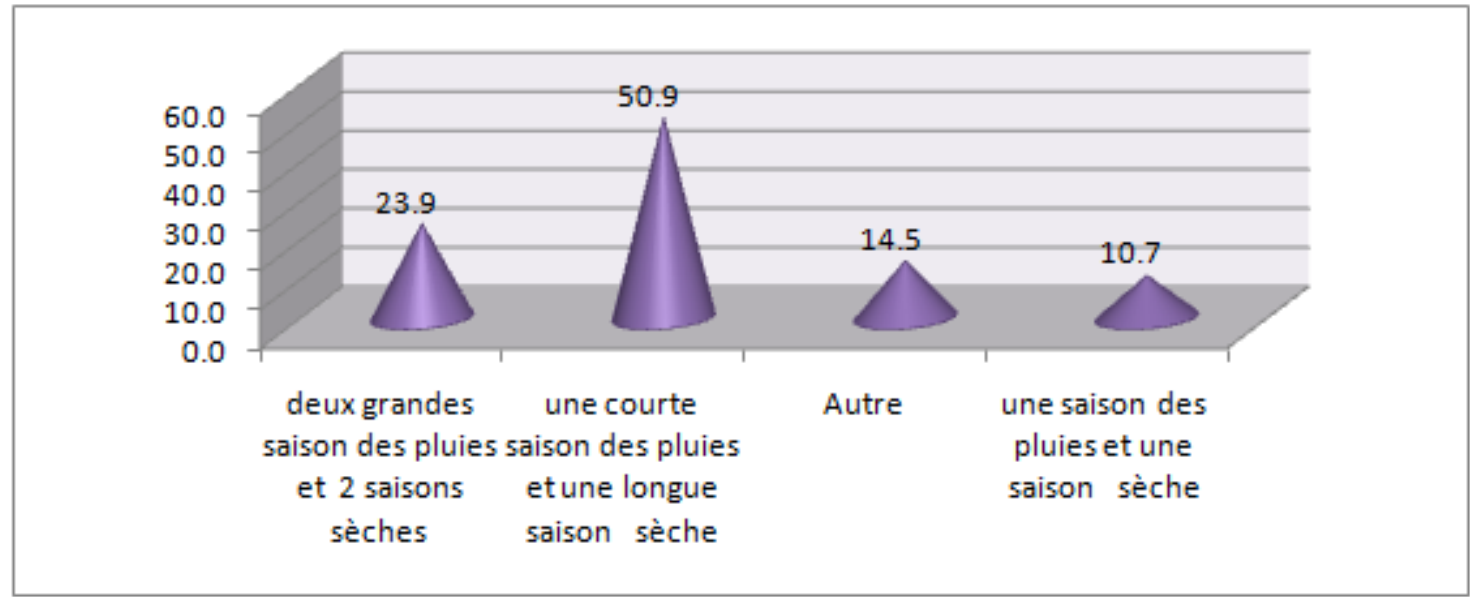

Source: Auteur à partir des données d'enquête.

S'agissant des différentes saisons observées dans la localité actuellement, on constate que sur 159 individus enquêtés, $23,9 \%$ énoncent «deux grandes saison des pluies et 2 saisons sèches»; 50,9\% énoncent «une courte saison des pluies et une longue saison sèche»; 14,5\% énoncent «autre »et $10,7 \%$ énoncent pour «une saison des pluies et une saison sèche». Il ressort qu'en majorité au sein de notre échantillon les différentes saisons observées dans la localité sont «une courte saison des pluies et une longue saison sèche».

\subsubsection{Influence Des Variations Annuelles Sur Des Performances Scolaires Des Elèves.}

Graphique : les variations annuelles des performances scolaires

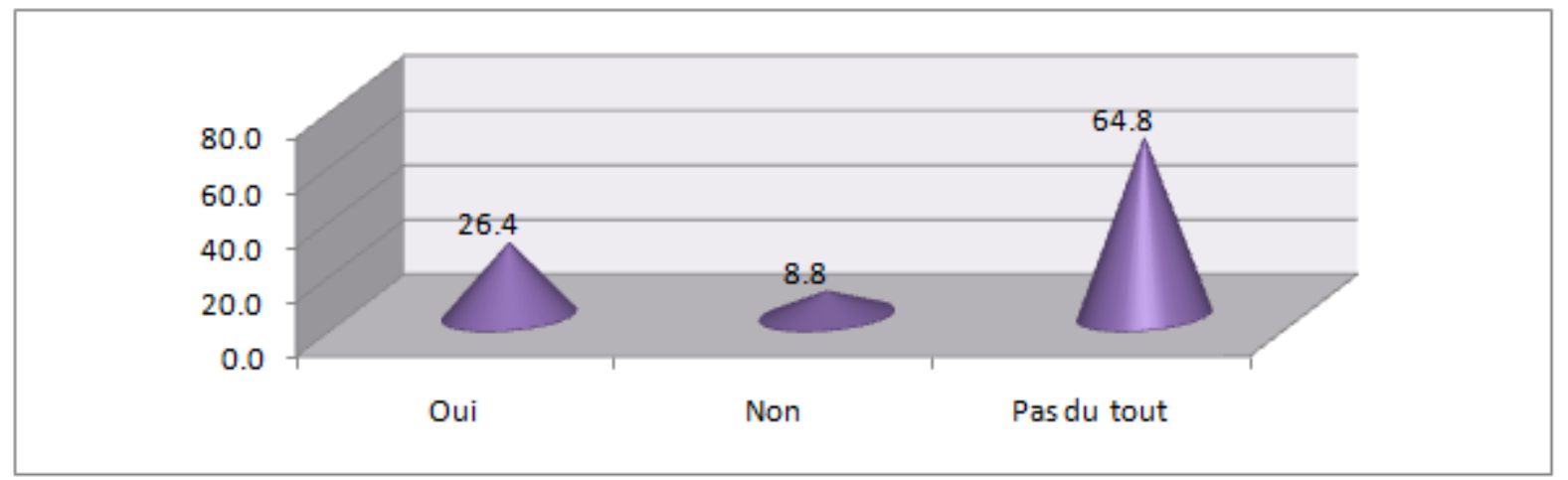

Source: Auteur à partir des données d'enquête.

A la question, Ces horaires (7h30-14h 30) vous facilite-t-il un rendement optimal, malgré les variations annuelles des performances scolaires de vos élèves et les données chrono biologiques ?, on constate que sur 159 individus enquêtés, 26,4\% répondent par «Oui »; 8,8\% répondent par «Non»; $64,8 \%$ répondent par «Pas du tout ». Il ressort qu'en majorité au sein de notre échantillon les horaires (7h30-14h 30) ne facilite pas du tout un rendement optimal, malgré les variations annuelles des performances scolaires de nos élèves.

5.1.3. Satisfaction de l'adoption des réformes locales en matière d'éducation.

Graphique : satisfait de l'adoption des réformes locales en matière d'éducation 


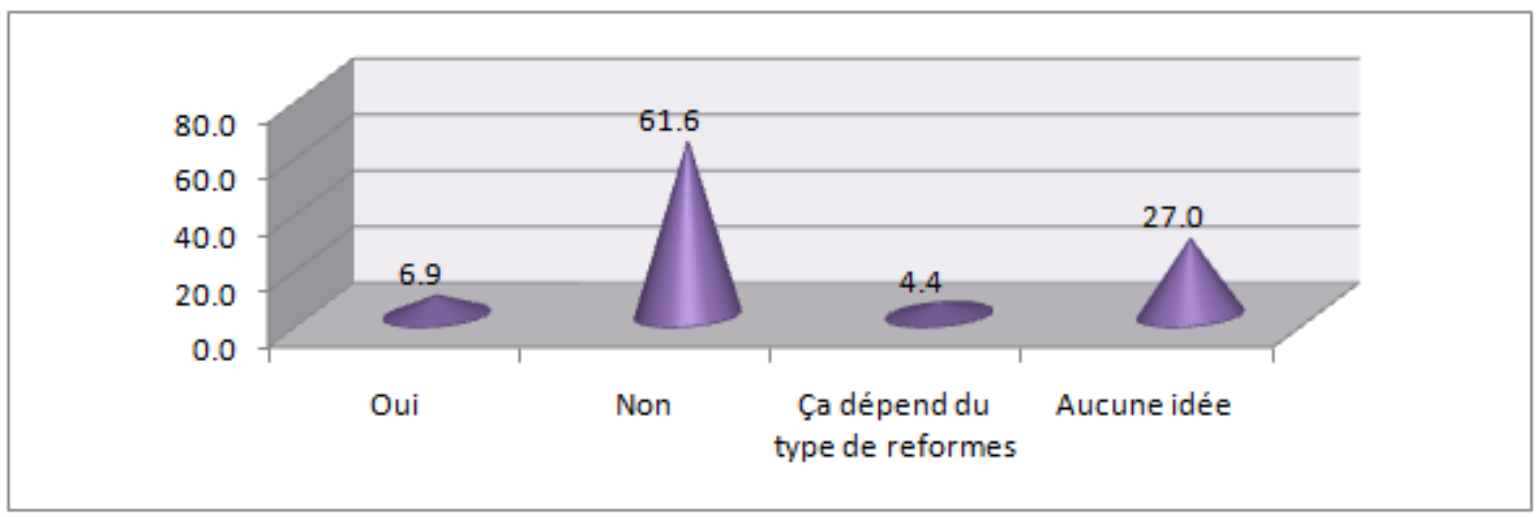

Source: Auteur à partir des données d'enquête.

À la question, Etes-vous satisfait de l'adoption des réformes locales en matière d'éducation dans la région de l'extrême nord actuellement?, on constate que sur 159 individus enquêtés, 6,9\% répondent par «Oui»; 61,6\% répondent par «Non»; 27,7\% répondent par «aucune idée». Il ressort qu'en majorité au sein de notre échantillon, les répondants/répondantes ne sont pas satisfaits de l'adoption des Réformes Locales En Matière D'éducation Dans La Région De l'Extrême-Nord Actuellement.

\subsubsection{Récapitulatifs De Certains Variables Des Conditions Climatiques Sévères}

\begin{tabular}{|l|l|l|}
\hline libellé & Oui & Non \\
\hline La saison sèche est-elle de plus en plus longue ? & $73 \%$ & $27 \%$ \\
\hline Fait-il de plus en plus chaud dans la journée pendant la saison sèche ? & $64,8 \%$ & $35,2 \%$ \\
\hline Les séquences sèches sont-elles de plus en plus longues ? & $63,5 \%$ & $36,5 \%$ \\
\hline Les saisons des pluies sont-elles de + en + pluvieuses (précipitation)? & $35,2 \%$ & $64,8 \%$ \\
\hline Les vents violents sont-ils de + en + fréquents pendant la saison des pluies ? & $37,1 \%$ & $62,9 \%$ \\
\hline
\end{tabular}

Source: Auteur à partir des données d'enquête.

À la question, La saison sèche est-elle de plus en plus longue ?, on constate que sur 159 individus enquêtés, $73,0 \%$ répondent par «oui»; 27,0\% répondent par «Non». Il ressort qu'en majorité au sein de notre échantillon, La saison sèche est de plus en plus longue. À la question, Fait-il de plus en plus chaud dans la journée pendant la saison sèche ?, on constate que sur 159 individus enquêtés, 64,8\% répondent par «oui»; 35,2\% répondent par «Non». Il ressort qu'en majorité au sein de notre échantillon, il fait de plus en plus chaud dans la journée pendant la saison sèche.

À la question, les séquences sèches sont-elles de plus en plus longues ?, on constate que sur 159 individus enquêtés, 63,5\% répondent par «oui»; 36,5\% répondent par «Non». Il ressort qu'en majorité au sein de notre échantillon, Les séquences sèches sont de plus en plus longues. À la question, les saisons des pluies sont-elles de + en + pluvieuses (précipitation) ?, on constate que sur 159 individus enquêtés, 35,2\% répondent par «oui»; 64,8\% répondent par «Non». Il ressort qu'en majorité au sein de notre échantillon, les saisons des pluies ne sont pas de + en + pluvieuses (précipitation).

À la question, Les vents violents sont-ils de + en + fréquents pendant la saison des pluies?, on constate que sur 159 individus enquêtés, $37,1 \%$ répondent par «oui»; 62,9\% répondent par «Non». Il ressort qu'en majorité au sein de notre échantillon, les vents violents ne sont pas de + en + fréquents pendant la saison des pluies.

\subsection{Analyse Bi Variée De Quelques Résultats De L'enquête}

Il est question ici de présenter le Profil entre le sexe et la planification des temps et rythmes scolaires des élèves de la région de l'Extrême-Nord (5.2.1.), Profil entre satisfaction de l'adoption des réformes locales en matière d'éducation et la planification des temps et rythmes scolaires des élèves de la région de l'Extrême-Nord (5.2.2.).

\subsubsection{Profil Entre Le Sexe Et La Planification Des Temps Scolaires Des Elèves De La Région De l'Extrême-Nord.}

Tableau: Profil entre le sexe et la planification des temps scolaires des élèves de la région de 
l'Extrême-Nord.

\begin{tabular}{|c|c|c|c|}
\hline & & \multicolumn{2}{|c|}{ Sexe du répondant/répondante } \\
\hline & & Masculin & Féminin \\
\hline \multirow{5}{*}{$\begin{array}{l}\text { laplanification des temps et } \\
\text { rythmesscolaires des élèves de la région de } \\
\text { l'Extrême-Nord. }\end{array}$} & Pas bienPlanifié & $0,0 \%$ & $0,0 \%$ \\
\hline & PeuPlanifié & $25,2 \%$ & $13,2 \%$ \\
\hline & Passable & $29,6 \%$ & $16,4 \%$ \\
\hline & Bien Planifié & $6,3 \%$ & $3,1 \%$ \\
\hline & TrèsbienPlanifié & $2,5 \%$ & $3,8 \%$ \\
\hline
\end{tabular}

Source: Auteur à partir des données d'enquête.

Parmi les de répondants/répondantes qui énoncent que la planification des temps scolaires des élèves de la région de l'Extrême-Nord est peu planifié, 25,2\% sont de sexe masculin et 13,2\% sont de sexe féminin (ligne «Peu Planifié »; colonne « Masculin» et «féminin» les pourcentages 25,2\% et 13,2\% $\%$ respectivement. Egalement parmi ceux qui énoncent que la planification des temps et rythmes scolaires des élèves de la région de l'extrême Nord est Passable, 29,6\% sont de sexe masculin et $16,4 \%$ sont de sexe féminin (ligne «assez bonne» colonne «Masculin») et «féminin» les pourcentages $29,6 \%$ et $16,4 \%$ respectivement.

\subsubsection{Profil Entre Satisfaction De L'adoption Des Réformes Locales En Matière D'éducation Et La Planification Des Temps Scolaires Des Elèves De La Région De l'Extrême-Nord.}

Tableau: Profil entre satisfaction de l'adoption des réformes locales en matière d'éducation et la planification des temps scolaires des élèves de la région de l'extrême Nord.

\begin{tabular}{|c|c|c|c|c|c|}
\hline & \multicolumn{4}{|c|}{$\begin{array}{l}\text { Etes-voussatisfait de l'adoption des réformes locales en } \\
\text { matièred'éducationdans la région de l'Extrême-Nord } \\
\text { actuellement? }\end{array}$} \\
\hline & & Oui & Non & $\begin{array}{l}\text { Çadépend du type } \\
\text { de reformes }\end{array}$ & Aucune idée \\
\hline \multirow{5}{*}{$\begin{array}{l}\text { laplanification des temps et } \\
\text { rythmesscolaires des élèves } \\
\text { de la région de l'Extrême- } \\
\text { Nord. }\end{array}$} & Pas bienPlanifié & $0,0 \%$ & $0,0 \%$ & $0,0 \%$ & $0,0 \%$ \\
\hline & PeuPlanifié & $3,1 \%$ & $25,8 \%$ & $1,3 \%$ & $8,2 \%$ \\
\hline & Passable & $2,5 \%$ & $28,3 \%$ & $1,9 \%$ & $13,2 \%$ \\
\hline & Bien Planifié & $1,3 \%$ & $4,4 \%$ &, $6 \%$ & $3,1 \%$ \\
\hline & TrèsbienPlanifié & $0,0 \%$ & $3,1 \%$ &, $6 \%$ & $2,5 \%$ \\
\hline
\end{tabular}

Source: Auteur à partir des données d'enquête.

Parmi les de répondants/répondantes qui énoncent que la planification des temps et rythmes scolaires des élèves de la région de l'extrême Nord est peu planifié, 25,8\% répondent par «Non » à la question, Etes-vous satisfait de l'adoption des réformes locales en matière d'éducation dans la région de l'extrême nord actuellement? (ligne «Peu Planifié »; colonne «Non» le pourcentage 25,8\%). Egalement Parmi les de répondants/répondantes qui énoncent que la planification des temps et rythmes scolaires des élèves de la région de l'extrême Nord est passable, 28,3\% répondent par « Non » à la question, Etes-vous satisfait de l'adoption des réformes locales en matière d'éducation dans la région de l'Extrême-Nord actuellement? (ligne «passable»; colonne « Non» le pourcentage $28,3 \%)$.

\section{Discussion Et APPORTS Des Resultats De L'ETUDE QuANTITATIVE.}

Il est question de présenter le récapitulatif des résultats de l'étude quantitative puis les interpréter.

Tableau: récapitulatif des résultats.

\begin{tabular}{|l|l|l|l|l|l|l|}
\hline Hypothèses & $\alpha$ & Sig-R & STUDENT calculé & STUDENT lue & R & Décision \\
\hline HR1 & 0,05 & 0,000 & $-3,964$ & 1,96 & $-0,303$ & confirmée \\
\hline HR2 & 0,05 & 0,000 & $-3,739$ & 1,96 & $-0,286$ & confirmée \\
\hline HR3 & 0,05 & 0,000 & $-3,842$ & 1,96 & $-0,293$ & confirmée \\
\hline HR4 & 0,05 & 0,000 & $-4,214$ & 1,96 & $-0,319$ & confirmée \\
\hline HR5 & 0,05 & 0,000 & $-3,982$ & 1,96 & $-0,303$ & confirmée \\
\hline HR6 & 0,05 & 0,000 & $-4,250$ & 1,96 & $-0,321$ & confirmée \\
\hline
\end{tabular}

Source: auteur de la recherche. 
Etant donné que Sig-R $=0,000<0,05$ ou encore que $t_{c a l} \succ t_{l u}^{\alpha / 2}(n-2)$, c'est-à-dire 3,964>1,96, alors H0 est rejetée et Ha est acceptée selon laquelle, il y a un lien significatif entre les conditions climatiques sévères suivant les périodes des années scolaire (mi-mars-mi-mai) et la planification des temps scolaires sur les performances des élèves de la région de l'extrême Nord et on constate au vu du tableau des résultats ci-dessus que $\mathrm{R}=-0,302<0$, alors on peut conclure que les conditions climatiques sévères suivant les périodes des années scolaire (mi-mars-mi-mai) influencent les temps et rythmes scolaires des élèves de la région de l'Extrême- Nord, Ce qui confirme H1.

Ces résultats confirment les conclusions des travaux deFrancoisTestu (2008); en effet, La chrono psychologie en milieu scolaire montre que les capacités de vigilance et de concentration évoluent avec l'âge. Les capacités de concentration, par jour, en terme de temps, sur des activités scolaires proprement dites sont de l'ordre de 3 heures pour les 6-8 ans, de 4 heures pour les 8-10 ans et de 5 heures de 10 à 11 ans $^{1}$. Les fluctuations journalières évoluent tant au niveau quantitatif que qualitatif comme nous l'explique François Testu : «Non seulement les scores bruts aux tests psychotechniques, les comportements d'écoute en classe, mais également les stratégies de traitement de l'information fluctuent au cours de la journée $»^{2}$.

Des études sur les fluctuations périodiques de certaines variables comportementales et physiologiques chez l'élève permettent de tirer quelques conclusions. Le début d'après-midi et, à un moindre degré, le début de matinée constituent deux moments «critiques» de l'évolution journalière de plusieurs variables biologiques (durée du sommeil diurne, rythme cardiaque, pression artérielle). Parallèlement, les performances mentales suivent les mêmes fluctuations.

François Testu et son équipe abordent dans ce sens : il existe deux périodes de plus faibles performances en début de demi-journée ( $8 \mathrm{~h} 30-9 \mathrm{~h}$ et $14 \mathrm{~h}-14 \mathrm{~h} 30)$ et deux périodes de plus fortes performances en fin de demi-journée (11h-11h30 et 16h-17h). Les performances ne remontent pas en fin d'après-midi autant qu'en fin de matinée même si plus l'enfant grandit, plus le pic du niveau de performance de l'après-midi ne se rapproche du niveau du matin. Les performances sont moins bonnes en début de matinée : la transition entre le sommeil et l'éveil complet passe par une phase appelée " inertie du sommeil », immédiatement consécutive au réveil et se caractérisant par une hypovigilance transitoire, des troubles de l'humeur et par une dégradation momentanée des performances physiques et mentales (troubles de la mémoire, des repères spatio-temporels, etc.). L'inertie se dissipe lentement de façon exponentielle.

De même étant donné que Sig-R $=0,000<0,05$ ou encore que $t_{c a l} \succ t_{l u}^{\alpha / 2}(n-2)$, c'est-à-dire 3,739> 1,96, alors $\mathrm{HO}$ est rejetée et Ha est acceptée selon laquelle, il y a un lien significatif entre les variations annuelles des performances scolaires des élèves du primaire(au 2ème et 3ème trimestre), et la rudesse climatique de la région de l'extrême Nord, et on constate au vu du tableau des résultats cidessus que $\mathrm{R}=-0,286<0$, alors on peut conclure que les variations annuelles des performances scolaires des élèves (au 2ème et 3ème trimestre), et la rudesse climatique influencent le temps et rythmes scolaires des élèves du primaire dans la région de l'Extrême Nord, Ce qui confirme H2.

Ces résultats confirment les conclusions des travaux deFraisse (1980); en effet, Selon Fraisse (1980) les comportements humains sont très dépendants des rythmes biologiques, mais les rythmes du comportement ne peuvent pas être seulement expliqués par les rythmes biologiques. Il est alors nécessaire d'étudier les rythmes du comportement pour eux-mêmes. Ainsi, la chronopsychologie est une discipline qui s'intéresse aux variations de comportements dans le temps, et particulièrement aux fluctuations journalières des performances intellectuelles. Les travaux en chronopsychologie ont permis d'établir des profils journaliers de performance en fonction de la nature des tâches cognitives, notamment dans le milieu scolaire (voir Testu, 2008).

Ainsi par exemple, Testu (1986) a repris le protocole de Shiffrin et Schneider (1977) en postulant qu'une tâche sous-tendue par un traitement contrôlé serait susceptible de donner lieu à une

\footnotetext{
${ }^{1}$ TESTU François, Rythmes de vie et rythmes scolaires, Aspects chrono-biologiques et chronopsychologiques, Paris, Masson, 2008.

${ }^{2}$ TESTU François, Rythmes de vie et rythmes scolaires, Aspects chrono-biologiques et chronopsychologiques, Paris, Masson, 2008.
} 
performance fluctuant au cours de la journée, ce qui ne serait en revanche pas le cas d'une tâche soustendue par un traitement automatique, pour laquelle la performance resterait stable. Ce protocole a été

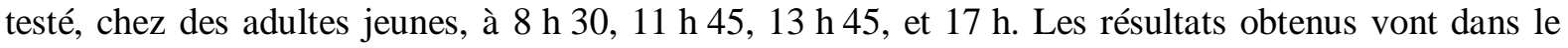
sens de l'hypothèse proposée, dans la mesure où la performance observée en traitement automatique ne fluctue pas au cours de la journée, tandis qu'elle varie dans la condition de traitement contrôlé (avec notamment deux pics de performance, l'un à $11 \mathrm{~h} 45$ et l'autre à $17 \mathrm{~h}$ ).

De même; Etant donné que Sig-R $=0,000<0,05$ ou encore que $t_{c a l} \succ t_{l u}^{\alpha / 2}(n-2)$, c'est-à-dire 3,842> 1,96, alors H0 est rejetée et Ha est acceptée selon laquelle, il y a un lien significatif entre les efforts déployés par le personnel enseignant, les données chonopsychologiques et la planification des temps et rythmes scolaires des élèves de la région de l'extrême Nord et on constate au vu du tableau des résultats ci-dessus que $\mathrm{R}=-0,293<0$, alors on peut conclure malgré les efforts déployés par le personnel enseignant, les données chonopsychologiques influencent les temps et rythmes scolaires des élèves du primaire dans la région de l'Extrême Nord. Ce qui confirme H3.

Ces résultats se rapprochent des conclusions des travaux des auteurs de la théorie de «l'effet-maître»; en effet pour ces auteurs, du point de vue théorique, les performances des élèves varient d'un enseignant à l'autre ou d'un groupe d'enseignants à l'autre. Ces différences de performance semblent s'expliquer par la différence des niveaux de la qualification de l'enseignant, la méthode pédagogique appliquée et ses expériences. La qualification de l'enseignant réfère à sa formation académique tant au niveau disciplinaire qu'au niveau pédagogique.

En effet, Bressoux (2006) fait une classification des enseignants en deux catégories. Les enseignants efficaces et peu efficaces. Selon l'auteur, les enseignants peu efficaces, négligent les élèves faibles et ces derniers sont souvent l'objet de critique. Au lieu d'aider les élèves en difficulté d'apprentissage, les enseignants peu efficaces préfèrent diminuer le contenu du programme et s'en tenir uniquement aux éléments simples. Ils n'incitent pas ces catégories d'élèves aux exercices de réflexion. Bressoux (2006) a fait remarquer que le jugement des maîtres joue aussi un grand rôle dans la performance scolaire, surtout chez les filles. Lorsqu'un enseignant développe des stéréotypes sexués à l'endroit des élèves, la progression de ces derniers peut être affectée très fortement.

Les enseignants efficaces donnent régulièrement des devoirs de maison aux élèves et qu'ils corrigent en classe. Ils voient toujours les contenus des programmes pédagogiques prévus. Ils organisent de petites évaluations formatives de manière périodique afin de pouvoir ajuster les apprentissages.

De même; Etant donné que Sig-R $=0,000<0,05$ ou encore que $t_{c a l} \succ t_{l u}^{\alpha / 2}(n-2)$, c'est-à-dire 4,214> 1,96, alors H0 est rejetée et Ha est acceptée selon laquelle, il y a un lien significatif entre la mauvaise gestion des activités péri et extrascolaires et la planification des temps et rythmes scolaires des élèves de la région de l'Extrême-Nord et on constate au vu du tableau des résultats ci-dessus que $\mathrm{R}=-0,319<0$, alors on peut conclure que la mauvaise gestion des activités péri et extrascolaires a une influence sur les temps et rythmes scolaires des élèves du primaire dans la région de l'Extrême-Nord, Ce qui confirme $\mathrm{H} 4$.

Ces résultats se rapprochent des conclusions des travaux de Jean-Jacques Ducret(2010) à Genève ; en effet, Dans un rapport sur l'aménagement du temps scolaire et extra-scolaire réalisé en 2010 par le service éducation du canton de Genève, qui s'interroge sur la meilleure organisation à mettre en place à l'école à partir de plusieurs propositions étudiées, Jean-Jacques Ducret réalise une revue critique sur les recherches en chronobiologie et chronopsychologie en lien avec le temps scolaire. Il écrit : «une série d'études [...] ont porté aussi bien sur la question de l'existence d'une rythmicité propre aux enfants et aux adolescents que sur celle de l'effet d'aménagements scolaires différents sur la rythmicité standard, biologiquement ancrée, à supposer naturellement qu'une telle rythmicité ait préalablement pu être mise en évidence chez les enfants et les adolescents.

Etant donné que Sig-R $=0,000<0,05$ ou encore que $t_{c a l} \succ t_{l u}^{\alpha / 2}(n-2)$, c'est-à-dire 3,982>1,96, alors H0 est rejetée et Ha est acceptée selon laquelle, il y a un lien significatif entre une mauvaise élaboration du calendrier scolaire sur les données chronobiologiques, chronopsychologiques et climatiques et la planification des temps et rythmes scolaires des élèves de la région de l'extrême 
Nord et on constate au vu du tableau des résultats ci-dessus que $\mathrm{R}=-0,303<0$, alors on peut conclure que une mauvaise élaboration du calendrier scolaire sur les données chronobiologiques, chrono psychologiques et climatiques a une influence sur les temps et rythmes scolaires des élèves du primaire dans la région de l'Extrême Nord. Ce qui confirme H5.

Ces résultats confirment les conclusions des auteurs de la théorie incrémentaliste, en effet, ces auteurs s'inscrivant dans la théorie incrémentaliste ont été les premiers à mettre en cause le caractère anticipé des choix décisionnels en évoquant notamment la rationalité limitée des décideurs (Simon, 1955) et les aléas qui entourent la mise en œuvre des décisions complexes (Lindbloom, 1959; Quinn, 1982). Bien que ce courant constitue une avancée théorique dans la conception des visées de la planification, il n'apporte pas d'explication satisfaisante au comportement organisationnel observé, à savoir l'élaboration des plans d'éducation sur la base du format rationaliste alors que ces plans ne sont pas du tout utilisés par les instances techniques comme des instruments guidant l'exécution des décisions. En effet, dans la conception incrémentaliste, les aléas de l'action induisent des difficultés pour anticiper les réalisations futures et imposent ainsi à l'organisation de revoir à la baisse les ambitions de prévision rationnelle. L'organisation opère plutôt de manière itérative et clarifie progressivement les objectifs au fur et à mesure que la visibilité sur l'avenir s'améliore (Lindbloom, 1968). Or, dans notre cas de figure, il nous a semblé que la clarification des objectifs ne causait pas de problème aux planificateurs.

De même, Etant donné que Sig-R $=0,000<0,05$ ou encore que $t_{c a l} \succ t_{l u}^{\alpha / 2}(n-2)$, c'est-à-dire 4,250> 1,96, alors $\mathrm{H} 0$ est rejetée et Ha est acceptée selon laquelle, il y a un lien significatif entre une mauvaise prévision de la décentralisation en matière des réformes locales et la planification des temps scolaires des élèves de la région de l'Extrême-Nord et on constate au vu du tableau des résultats ci-dessus que $\mathrm{R}=-0,321<0$, alors on peut conclure que une mauvaise prévision de la décentralisation en matière des réformes locales a une influence sur les temps et rythmes scolaires des élèves du primaire dans la région de l'Extrême-Nord. Ce qui confirme H6.

Ces résultats se rapprochent des conclusions des travaux d'Hubert Montagner (1996); en effet, Hubert Montagner souligne l'importance de respecter le rythme veille sommeil : le temps de sommeil nocturne ainsi que le temps de sieste des plus petits préparent «l'enfant a la mobilisation de toutes ses compétences et de ses ressources intellectuelles. Réparateur[s] aux plans biologique et psychologique, [ils participent] aussi à la consolidation des acquis maitrises lors des temps d'éveil ». Les perturbations de ce rythme veille-sommeil peuvent donc avoir des conséquences négatives sur " le niveau de vigilance, les capacités d'attention et l'ensemble des compétences-socles de l'enfant $»^{3}$ Le respect de ce rythme est complexe car il peut être très différent d'une personne à une autre. L'acquisition du rythme veille-sommeil ne se fait pas pour tous les enfants au même moment de leur enfance. Les durées de sommeil varient selon l'âge de l'enfant : un enfant de Grande Section dort en moyenne 653 minutes par nuit alors qu'un enfant de CM2 dort 610 minutes $^{4}$.

Les durées de sommeil varient également selon si l'enfant est un gros dormeur ou un petit dormeur. Montagner cite l'exemple d'enfants de 4 ans : dans des conditions leur permettant de dormir selon leurs besoins, les petits dormeurs dorment de 7 à 8 heures alors que les gros dormeurs peuvent dormir entre 13 et 14 heures. Montagner rappelle par la suite que même si un certain nombre d'enfant se retrouvent dans les catégories petites ou gros dormeurs, un grand nombre sont considérés comme des «moyens dormeurs ». Il est donc important de souligner que chacun a son propre rythme.

\section{CONCLUSION}

Compte tenu de l'inscription du thème dans la mouvance des interactions rythmiques, cet article revêt une connotation psychopédagogique et socioéducative. Ainsi, cette étude s'inscrit dans le domaine d'ajustement du temps et rythmes scolaire en tenant compte des éléments liés aux aléas climatiques et environnementaux des écoliers du primaire. Comme nous le savons, nombreux sont les facteurs qui influencent les résultats des élèves. Cependant, nous ne pouvons prétendre les analyser tous. Donc cet

\footnotetext{
${ }^{3}$ MONTAGNER Hubert, En finir avec l'échec à l'école, L'enfant : ses compétences et ses rythmes, Paris, Bayard éditions, 1996,.p.156 et 158.

${ }^{4}$ TESTU François, Rythmes de vie et rythmes scolaires, Aspects chrono-biologiques et chronopsychologiques, Paris, Masson, 2008.
} 
article cherche uniquement à voir si l'organisation du temps scolaire en tenant compte des rythmes des enfants selon les spécificités climatiques sur les plans chronobiologique, chronopsychologique, chronosociologique et managérial auront une amélioration sur leur parcours scolaires. Etant donné que cet environnement est en constante évolution avec des perturbations climatiques qui sont perceptibles par un décalage constant des mois de sécheresse toutes ces dernières années dans la région de l'Extrême-Nord, il est important pour tous les organiseurs des systèmes éducatifs afin qu'aux travers des réformes, ils assurent aux élèves une planification des temps et rythmes scolaires adéquate. Autrement dit, avec les changements galopants liés à la mondialisation, les enjeux et défis majeurs des différentes sociétés sont élaborés en vue d'améliorer la qualité de l'éducation. Ceci passe par une organisation en tenant compte de certaines réalités (pédagogique, psychologique, biologique, sociologique). Et l'organisation des temps et rythmes scolaire étant une discipline scientifique doit à tout moment s'arrimer à cette donne. Cette organisation passe non seulement par la redéfinition des finalités, mais aussi par l'atteinte des objectifs plus équitable et plus efficace. Plus généralement, tout reste à faire au Cameroun, et ailleurs, pour que le temps scolaire soit d'abord organisé à partir des réalités constituées par les rythmes des enfants, les idéologies, les intérêts contradictoires des adultes et les lobbies politiques, économiques, religieux. Les données disponibles permettent d'apprécier des caractéristiques sur :

- La qualité et la quantité totale du temps scolaire selon les âges et le cycle scolaire mesurée en nombre d'an par an et selon les spécificités climatiques et environnementaux ;

- L'étalement de ce temps sur le calendrier annuel, à travers le nombre de semaine et de jours par trimestre ;

- La charge hebdomadaire et quotidienne : nombre de jours d'école par semaine, durée moyenne de la semaine scolaire et de la journée d'école et durée des séquences d'enseignement.

La combinaison de ces différentes variables donne une première image des rythmes propres à chaque organisation.

Les Suggestions, apports et recommandations de notre recherche se présentent ainsi qu'il suit :

\section{$>$ Suggestion de l'étude.}

Il faut prendre en considération l'apport des rythmes biologiques en attirant l'attention sur certains éléments importants comme :

* Instituer au moins une heure d'étude surveillée à la fin des enseignements de tous les après-midi.

\# Le sommeil : il faut que les parents veillent à ce que tous les enfants dorment suffisamment pour pouvoir récupérer l'énergie perdue dans la journée d'école.

* La vie à l'école : il faudrait tenter de diminuer le stress de l'enfant et le surmenage scolaire par des programmes adaptés et non pléthoriques en des périodes des hautes chaleurs ;

$>$ Eviter le transport des cartables lourds par les élèves en mettant à leur disposition des cantines et casiers pour la garde de leurs effets scolaires.

\section{$>$ recommandation de l'étude}

* Encourager la recherche en matière de planification des temps et rythmes scolaires, appuyer à ce sujet la création de liens et d'une coopération active à tous les niveaux (national, régional, sous régional, et international), entre les chercheurs activement engagés dans des programmes de recherche et de formation à tous ces niveaux là c'est-à-dire au niveau international, et aussi la création de réseau de coopération et de lien au niveau national, régional, sous régional.

Entreprendre des recherches sur la coopération interuniversitaire avec un accent particulier sur la planification des temps et rythmes scolaires, la gestion et l'exécution que cette coopération implique.

Les caractéristiques de l'environnement dépendent de sa situation géographique. Le lieu où nous vivons est une portion de la surface de la terre appartenant à une zone climatique. Alors, comment réussir les nouveaux ajustements de planification des temps et rythmes scolaires dans la région de l'Extrême-Nord? 
Plusieurs priorités se dégagent. La plus importante est de respecter les rythmes biologiques et psychologiques des enfants en allégeant le temps scolaire sur la journée, notamment pour les plus jeunes : réserver les moments reconnus comme favorables à l'activité intellectuelle (début de la matinée pour ceux qui pratiquent la journée à plein temps pour les apprentissages et les activités demandant le plus d'attention et d'effort cognitif ; occuper les moments les moins favorables par des activités d'entretien, d'éveil, des contenus plus ludiques et socialisants ; moduler les horaires et les durées de classe en fonction de l'âge ; ajuster des structures pour accueillir les élèves avant et après la classe, et durant le creux de vigilance d'après-déjeuner, où les activités non scolaires, tout aussi éducatives mais moins fatigantes, seraient encadrées par les éducateurs.

Enfin, le réajustement du temps scolaire impose une réflexion sur le rôle de l'école et la répartition des responsabilités éducatives entre enseignants, parents et responsables des mouvements associatifs.

\section{REFERENCES BIBLIOGRAPHIQUES.}

[1] Bressoux, P., \& Pansu, P. (2003). Quand les enseignants jugent les élèves. Paris: P. U. F,

[2] Bonnery, S. (2006). Comprendre l'échec scolaire, Élèves en difficultés et dispositifs pédagogiques la Dispute, 256 p.

[3] Bryman, A. (2006). Integrating Quantitative and Qualitative Research: How Is It Done? Qualitative Research, 6, 97-113. http://dx.doi.org/10.1177/1468794106058877

[4] CoutyP.(1984). Statistique et recherches interdisciplinaires: Implication d'une discipline sans objet.

[5] Cermakian, N., Whitmore, D., Foulkes, N.S., and Sassone-Corsi, P. (2000). Source: Journal of molecularmedicine (Berlin, Germany) 78(7): B46 (Abstract)

[6] Fraisse P. (1975). Psychologie du temps, Paris, PUF.

[7] Fraisse, P. (1967). Psychologie du temps. Paris : PUF, (2ème édition).

[8] Fraisse, P. (1970).L'évolution de la psychologie expérimentale. In: Traité de psychologie expérimentale. Fraisse, P., \& Piaget, J. Paris : PUF, 5-79.

[9] Fraisse, P. (1980). Eléments de chronopsychologie, Le travail humain. 43, 2, 353-372.

[10] Ducret, J-J. (2010). Aménagement du temps scolaire et extrascolaire : vers un nouvel horaire scolaire, ed Genève SRED 2010. Collection Document / SRED, 10.005.

[11] Lindbloom, (1959).The Science of "MuddlingThrough": Public Administration Review, Vol. 19, No. 2 (Spring, 1959), pp. 79-88Published by: BlackwellPublishing on behalf of the American Society for PublicAdministrationStable URL: http://www.jstor.org/stable/973677

[12] Montagner, H. (1996).En finir avec l'échec à l'école, L'enfant : ses compétences et ses rythmes, Paris, Bayard editions, 283p.

[13] Montagner, H. (1983). Les rythmes de l'enfant et de l'adolescent : ces jeunes en mal de temps et d'espace, Paris, Stock-Laurence Pernoud, ,445 p.

[14] Montagner, H. (2006). La sécurité affective, la socialisation, les rythmes, l'aménagement des temps et des espaces, dans la conférence Le développement et l'éducation du jeune enfant. L'importance de l'école.

[15] Perrenoud, P. (1984). La fabrication de l'excellence scolaire. Genève, Droz.

[16] Quinn, (1982). Magnetic Orientation in Animals ; publié dans la revue Springer Shop\&Amazon Francepar De RoswithaWiltschko

[17] Shiffrin, RM., \& Schneider W. (1977). Controlled and automatichuman information processing: II. Perceptuallearning, automatic attention and a generaltheory. PsychologicalReview, 84, 127-190

[18] Testu, F. (1989). Chrono-psychologie et rythmes scolaires, Paris, Masson, 120p.

[19] Testu, F. (2008).Rythmes de vie et rythmes scolaires, Aspects chrono biologiques et chrono psychologiques, Paris, Masson, 149p.

[20] Touitou, Y., \&Haus, E. (1994). Biologicrhythms and laboratorymedicine. New-York: Springer- Verlag, 2ème edition.

[21] Touitou, Y., Bogdan, A., Haus, E., \&Touitou, C. (1997). Modifications of circadian and circannualrhythmwithaging. Experimentalgerontology, 32, 693-614.

[22] Touitou, Y., Bogdan, A., Auzeby, A., \&Selmaoui, E. (1998).Mélatonine et vieillissement. Thérapie, 53, 473-478.

[23] Touitou, Y. (1999). Rythmes de vie chez l'enfant. Archives de Pédiatrie, 6, 289-291.

[24] Tardif et Lessard. (1999). Le travail enseignant au quotidien : contribution à l'étude du travail dans les métiers et les professions d'interactions humaines, Québec, Presse de l'université de Laval. 
[25] Winter. (1984). "For Better or Worse": Attitudes TowardMarriage in Literature (Part I) An InterdisciplinaryCritical Journal Vol. 17, No. 1.

\section{AUTHOR'S BIOGRAPHIES}

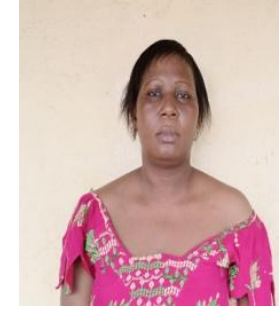

DJARA NDJIDDA est née le $1^{\text {er }}$ janvier 1987 à DANA, arrondissement Yagoua dans la région de l'Extrême-Nord Cameroun. Je suis issue d'une famille de classe moyenne.

Après mes études secondaires au lycée classique et moderne de Yagoua, je me suis directement inscrite à l'université de Yaoundé $\mathrm{I}$ où $\mathrm{j}$ 'ai décidé de poursuivre mes études supérieures à la Faculté des Arts, Lettres et Sciences Humaines (FALSH). J'ai obtenu mon Master I en histoire des Relations Internationales en 2013. En 2014, j'ai décidé de poursuivre mes études à la faculté des Sciences de l'Education où j'ai obtenu mon Master II en Management de l'Education.

Actuellement, je suis en cycle Doctorat Phd $3^{\text {ème }}$ année.

Citation: Djara Ndjidda. "Planification Du Temps Scolaire Sur Les Performances Des Élèves Du Primaire Dans L'extrême-Nord Du Cameroun" International Journal of Humanities Social Sciences and Education (IJHSSE), vol 7, no. 8, 2020, pp. 23-38. doi: https://doi.org/10.20431/2349-0381.0708004.

Copyright: () 2020 Authors. This is an open-access article distributed under the terms of the Creative Commons Attribution License, which permits unrestricted use, distribution, and reproduction in any medium, provided the original author and source are credited. 